\title{
An Investigation on the Impact of Implementing Visual Quantum Mechanics on Student Learning and Student Instructor Beliefs*
}

\author{
Lawrence T. Escalada \\ Department of Physics \\ University of Northern Iowa \\ Cedar Falls, Iowa
}

\begin{abstract}
Aspects of the Visual Quantum Mechanics instructional materials have been adapted and implemented into a university physical science course for pre-service elementary education majors and various high school physics classrooms. These materials utilize a learning cycle pedagogy involving interactive, simulation computer programs and inexpensive devices to introduce basic quantum physics ideas within the context of fundamental physics concepts. A brief description of how these materials and strategies were adapted and implemented in high school classrooms will be provided. The results found on student conceptual learning and student/instructor attitudes and beliefs will also be briefly discussed.

* Supported in part by NSF Grant \#ESI9730586 and a Iowa Space Grant Consortium Phase I - "Seed" Grant Introduction
\end{abstract}

As our standard of living becomes more dependent on the latest developments in science and technology, the literacy of our students must be at a level that enables them to maintain at least their parents' standard of living and make educated decisions on science- and technology-related issues as well as on the everyday applications of the latest developments in science and technology. Students need to be able to at least have a basic understanding of the physical phenomena associated with these applications and the fundamental $20^{\text {th }}$
Century physics principles that explain these phenomena in order to make informed decisions about them. Unfortunately, many physics teachers exclude presenting modern topics such as quantum mechanics in high school physics courses or spend very little time introducing these topics as a result of the traditional focus on such topics as mechanics and electricity.[1][2] The high degree of mathematical formalism and abstract nature associated with quantum mechanics also makes the subject extremely difficult to introduce in most high school physics courses.[3][4]

Visual Quantum Mechanics

Over the past few years the Kansas State Physics Education Research Group, under the direction of Dean Zollman, have been addressing these issues as part of the Visual Quantum Mechanics project. To enable a broad spectrum of students to learn quantum mechanics, a series of interactive instructional units have been developed that utilize hands-on activities and computer visualizations to introduce quantum principles to high school and introductory college students who have limited backgrounds in physics and mathematics. These instructional units utilize a modified learning cycle in which student investigations of concrete phenomena precede and follow the introduction of abstract concepts.[5] These instructional units include the following:

- Solids and Light - Students use their observations of the light 
emitted from solids and gases to help them understand why the energy in atoms is quantized and some of the consequences of that quantization as seen in everyday devices such as the light emitting diode (LED).[6]

- Luminescence: It's Cool Light! Students use their observations of the light emitted by luminescent materials to build energy level models to explain a variety of different light emitting processes.[7]

- Waves of Matter - Students explore the wave nature of matter by examining applications of quantum physics such as the electron microscope and the Star Trek Transporter through the use of visualization activities.[8]

- Potential Energy Diagrams Students are provided with classical experiences that enable them to understand the graphical displays of energy vs. distance which play an important role in understanding of the quantum ideas being introduced. [9]

\section{Solids \& Light}

Students begin by investigating the electrical properties of LEDS and comparing these properties with those of incandescent lamps. Students then observe the spectral properties of LEDs and compare these properties with those of gas and incandescent lamps. Students are then introduced to the energy level model of atom following these investigations as illustrated in Figure 1. The relationship between energy levels of an atom, electronic transitions (absorption and emission), and photons is also introduced.

Students then use an interactive computer program called Gas Lamp Emission Spectroscopy from the Spectroscopy Lab Suite software package to develop an energy level model of an atom of a gas that can explain the spectra emitted by gas lamps.[10] See Figure 2. Through the use of other simulation programs, students use this energy level model of an atom of a gas to build other models for atoms of solids that explain spectra emitted by LEDs and incandescent lamps. Students then use a computer program called LED Constructor to illustrate how energy bands of solids can be used to explain the electrical and spectral properties of LEDs.

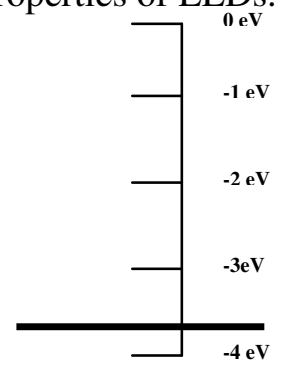

Figure 1: An Energy Diagram for an Electron with $-3.8 \mathrm{eV}$ of energy

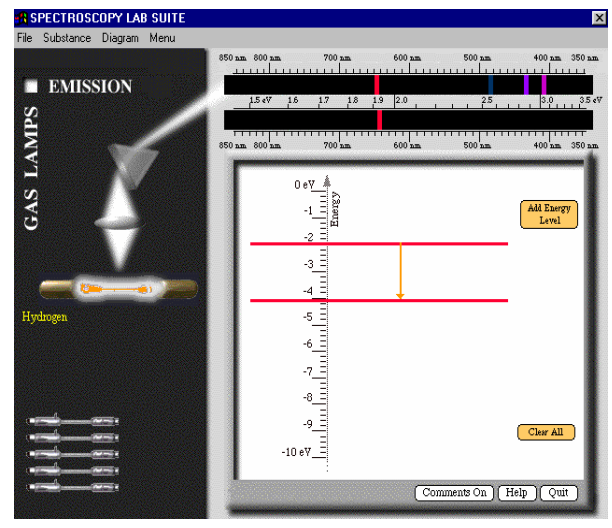

Figure 2: Gas Lamp Emission Spectroscopy Computer Program

\section{Luminescence: It's Cool Light!}

Students investigate the physical and spectral properties of various light sources. They are then introduced to the concept of the energy level model in a similar manner used in Solids. Students are also introduced to the concept of a potential energy diagram a visual graph of electrical potential energy vs. distance to represent a model 
of an atom. Students use an interactive computer program called Energy Band Creator to show how the placement and number of energy levels are affected by the depth and width of the potential energy diagrams, the number of diagrams, and the relative distance between diagrams. This program allows the student to visualize "atoms" of a gas, "atoms" of a solid, and "atoms" of a solid with impurities in terms of potential energy diagrams. By using this program, students develop an understanding how a band of energies result from adding a large number of impurity atoms to the solid. Students then use this idea in various computer programs to create an energy level model that explain the light properties of a fluorescent lamp, a phosphorescent toothbrush, a television monitor, and an infrared detection card.

\section{High School Implementation}

The Visual Quantum Mechanics materials have been extensively field tested in a number of different high school, college, and university physics classrooms. The results of some of these field tests involving high school physics classrooms indicate that the use of hands-on activities, interactive computer programs, inexpensive materials, and the focus on conceptual understanding are very applicable in the classrooms observed.[11] We found that teachers who are not familiar or comfortable with the topic and/or pedagogical intent of the materials will modify the intent of the teaching strategies of these materials. As a result, it was difficult to determine whether the difficulties found in implementing the materials were due to the contemporary topic being introduced or due to the instructional strategies being used. As a result, a second study was undertaken to investigate the impact of implementing Solids and/or Luminescence in various high school physics classrooms in which the teachers are already familiar and comfortable with the activity-based pedagogy. A variety of instruments including questionnaires, assessments, and an informal observation protocol were used to collect data.

Four Iowa high school teachers were selected and provided with training and support in implementing at Solids and/or Luminescence in their classrooms during the fall semester after mechanics was introduced. The teachers taught in a variety of high school environments whose enrollments ranged from 240 to 1250 students. The participating teachers had 7 to 25 years of teaching experience. All four teachers were experienced PRISMS (Physics Resources and Instructional Strategies to Motivate Students) teachers. The PRISMS materials contain high-interest activities related to real-life experiences of high school physics students.[12] The materials also utilize a learning cycle pedagogy and focus on the use on inexpensive resources. The participating students and teachers preferred and were comfortable with an activity-based learning environment.

Students indicated that they liked the hands-on nature of the activities, using computer programs to investigate the relationship between spectral patterns and energy levels, and the real-life science applications. When asked to indicate what they learned as a result of completing Solids and/or Luminescence, some students indicated the mechanism for emission and absorption of light - relating energy diagrams of atoms to spectra, different ways in which light can be emitted, and how potential energy diagrams can be used to represent all types of atoms from gases to solids.

When asked what difficulties were encountered, some students and 
teachers indicated that there was some difficulty with understanding the concept of a potential energy diagram used in Luminescence and the solidstate nature of LEDs found in Solids. Some students and teachers felt more background on LEDs and light was necessary. All four teachers indicated they would implement the materials again especially after the topics of mechanics, energy, and light were introduced.

Based on the results of student assessments, students were able to recognize the spectral features of various sources. They were also able to use the energy model of the atom to explain the spectra properties of various light sources especially for the gas lamp. Students, however, had some difficulties with applying the energy model of the atom to glow-in-dark and fluorescent objects. Students also had some difficulty with distinguishing between the emission and absorption of light in terms of electronic transitions.

\section{Conclusion}

In the high school classrooms studied, there are aspects of using the energy model of the atom that appear to be difficult for both the student and for the teacher. It appears that the students have a basic understanding of the energy model that can explain the spectral properties of a gas lamp. This could be due to both Solids and Luminescence providing students with numerous opportunities to revisit and use this model as a foundation for the energy models of other light sources. The students, however, appear to need additional experiences to use and discuss the various energy models that explain the spectral properties of solidstate light sources. Both students and their teachers would have benefited from the experiences in applying the energy vs distance graph to analyze classical scenarios found in the Potential Energy Diagrams unit. Unfortunately, the teachers and students involved in this study did not have access to this unit. With additional experiences with the energy models being introduced within the context of concrete experiences, it should be possible to introduce $20^{\text {th }}$ century physics ideas within the high school curriculum where it did not exist previously with enthusiasm, interest, and understanding.

\section{References}

[1] Hobson, A. "Teaching quantum theory in the introductory course", Phys. Teach., 34, 202-210 (1996).

[2] Neuschatz, M., \& Alpert, L. Overcoming Inertia: High School Physics in the 1990s (American Institute of Physics, Maryland, 1996).

[3] Johnston, I. D., Crawford, K., \& Fletcher, P. R. "Student difficulties in learning quantum mechanics", Int. Jrnl. Sci. Ed., 20, 427-446 (1998).

[4] Morgan, M. J., \& Jakovidis, G. "Characteristic energy scales of quantum systems", Phys. Teach. 32, 354-358 (1994).

[5] Karplus, R. "Science teaching and development of reasoning", Jrnl Res. Sci. Tching, 12, 213-217 (1974).

[6] Escalada, L. T., Rebello, N. S., \& Zollman, D. A. Solids \& Light: Explorations of Quantum Effects in Light Emitting Diodes (Kansas State University, Kansas, 2001)

[7] Escalada, L. T., Rebello, N. S., \& Zollman, D. A. Luminescence: It's Cool Light!: An Investigation on the Quantum Effects in Luminescent Materials and Devices Emitting Diodes (Kansas State University, Kansas, 2001)

[8] Rebello, N. S., \& Zollman, D. A. Waves of Matter (Kansas State University, Kansas 2001)

[9] Rebello, N. S., \& Zollman, D. A. Potential Energy Diagrams (Kansas State University, Kansas 2001)

[10] Rebello, N. S., Ravipati, C., Zollman, D. A., \& Escalada, L. T. "Simulating the spectra of light sources", Comp. Phys., 28, 33 (1998).

[11] Escalada, L. T. Investigating the Applicability of Activity-Based Quantum Mechanics in a Few High School Physics Classroom (Doctoral Dissertation, Kansas State University, 1997). University Microfilms No. 9804410.

[12] Unruh, R., Cooney, T., McGrail, D., \& et al. Physics Resources and Instructional Resources for Motivating Students (University of Northern Iowa, Iowa 1993). 
